\title{
Formularaufwand
}

\section{Binden Sie Patienten bei der Erledigung von Anträgen mit ein}

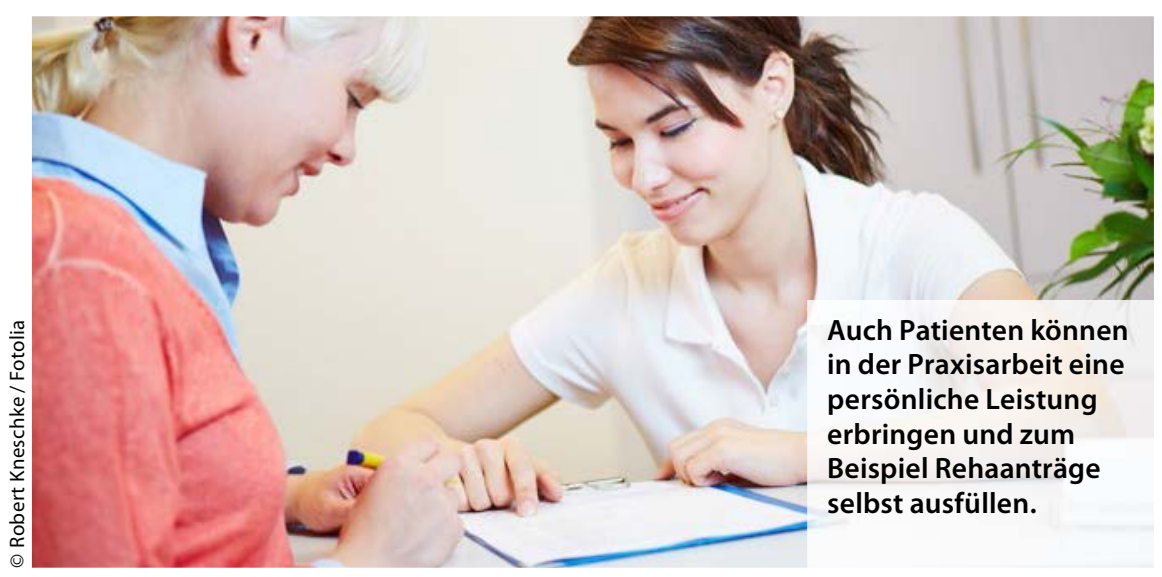

Die Delegation von geeigneten Aufgaben an Mitarbeiter stärkt die Teamorientierung in Arztpraxen. Dabei wird eines oft übersehen: Auch der Patient kann Teil des Teams werden und seinen Teil zum Prozess beitragen, zum Beispiel bei Formularen.

$\mathrm{P}$ atienten geben häufig Formulare wie Reha- oder Kuranträge in der Praxis ab, mit der Bitte diese für sie zu erledigen. Bürokratischer Aufwand entsteht aber beispielsweise auch durch unzählige Anfrageformulare der Krankenkassen. Da kommt schnell die Idee, diese Aufgaben zu delegieren, um den Aufwand zu reduzieren. Doch welche Aufgaben darf der Arzt delegieren beziehungsweise dürfen rechtlich gesehen delegiert werden? Und an welche Personen sind Leistungen und Aufgaben überhaupt delegierbar? Antworten auf diese Fragen finden sich nicht nur im SGB V, der Musterberufsordnung der Ärzte oder in Stellungnahmen von Kassenärztlicher Bundesvereinigung (KBV) und Bundesärztekammer (BÄK). Antworten finden sich auch in allgemeinen organisatorischen und betriebswirtschaftlichen Grundsätzen. Unter ande- rem gilt, dass alle Aufgaben, die ein Anderer besser oder zumindest gleichwertig erledigen kann, auf diesen übertragen werden können sollten.

\section{Betriebswirtschaftliche Grundsätze}

Bei der Erbringung ärztlicher Leistungen gilt in erster Linie die Verpflichtung der persönlichen Leistungserbringung. Werden ärztliche Leistungen auf einen anderen Arzt übertragen, ergeben sich nur geringe Probleme. Der delegierende Arzt kann darauf vertrauen, dass der andere Arzt die Leistungen mit der erforderlichen Qualität und Sorgfalt erbringt. Eine Delegation an einen Arzt, der nicht über eine zur Erbringung der Leistung erforderliche Abrechnungsgenehmigung oder fachliche Qualifikationsbescheinigung seiner Kassenärztlichen Vereinigung verfügt, ist dagegen - auch nach Rechtsprechung des Bundessozialgerichts - unzulässig.

Anders verhält es sich jedoch bei der Delegation von Leistungen an nicht ärztliche Mitarbeiter. Höchstpersönliche ärztliche Leistungen sind in diesem Falle zwar nicht delegierbar. Blutabnahmen, Injektionen und Infusionen sowie die Einlage von Blasenkathetern und so manche weitere Aufgabe mehr können jedoch von qualifizierten nicht ärztlichen Mitarbeitern ausgeführt werden. $\mathrm{Zu}$ diesen Aufgaben gehört beispielsweise auch das Ausfüllen von Anträgen und anderen Formularen. Dabei ist immer zu berücksichtigen, dass die grundlegende Intention nicht ist, die Arbeit auf andere abzuwälzen. Die Überlegung muss vielmehr darauf zielen, die Aufgaben demjenigen zu übertragen, der die höhere Kompetenz und Zuständigkeit dafür hat diese $\mathrm{zu}$ erledigen. Hier kommt der Patient ins Spiel. Fasst man den Begriff Delegation weit, können Aufgaben natürlich auch an Patienten übertragen werden. Denken Sie nur an die vielen Formulare oder auch Krankenkassenanfragen, die der Patient in Ihrer Praxis abgibt. Auf diesen fehlen häufig die persönlichen Angaben des Patienten und vieles mehr.

Es ist einfach, alles in der Praxis abzugeben. Doch solche Unterlagen, in denen die Teile, die vom Patienten zu erledigen sind - einschließlich der Angabe seines Namens - und von diesem nicht ausgefüllt wurden, können ganz freundlich postwendend zurückgegeben, also an den Patienten delegiert werden. Das ist nicht Aufgabe des Arztes und auch nicht die Aufgabe seiner Mitarbeiterinnen. Wer beim Delegieren nicht zu fordernd vorgeht, spart am Ende nicht nur Arbeit, er bindet auch den Patienten in den Prozess mit ein und gibt ihm ein Stück mehr Verantwortung.

Dr. Peter Schlüter 